\title{
Stokes velocity generated by a point force in various geometries $^{\star \star \star}$
}

\author{
Maria Ekiel-Jeżewska ${ }^{a}$, Robert Boniecki, Marek Bukowicki, and Marta Gruca \\ Institute of Fundamental Technological Research, Polish Academy of Sciences, Pawińskiego 5b, 02-106 Warsaw, Poland
}

Received 6 April 2018

Published online: 12 October 2018

(c) The Author(s) 2018. This article is published with open access at Springerlink.com

\begin{abstract}
In this short review, we visualize the fluid velocity generated by a point force close to a plane free surface or a plane rigid wall. We present separately contributions from all the multipoles which form the corresponding classical systems of images. Such graphical images might be useful in the theoretical and numerical modeling of the dynamics of micro-objects moving close to an interface.
\end{abstract}

\section{Introduction}

There has been recently a lot of interest in the dynamics of micro-objects of different shapes moving in a bounded geometry - close to a rigid wall, an interface between fluids or free surface [1-12]. The problem as to how the presence of boundaries affects swimming of microorganisms, such as bacteria, algae or sperm [11-27], motion of red blood cells [28, 29], Janus particles [30] or micro-robots [31,32], is important for biological, environmental, medical and industrial applications.

Importantly, the fluid velocity field around such moving objects as algae or bacteria can now be directly measured with a high accuracy [33,34]. Such experimental data allow for the development of efficient and simple theoretical and numerical models of the dynamics of such complex real micro-objects as, e.g., Chlamydomonas, and apply them to explain the transport properties of biological suspensions, e.g. the viscosity of flagella-beating algae [35].

The goal of this review is to provide a visualization of the velocity field of the fluid around a point force close to a flat free surface or a plane rigid wall, separately for all the contributing multipoles. The results can be used to construct models of micro-objects moving in confined geometries, based on a comparison with the experimentally measured fluid velocity.

\footnotetext{
* Contribution to the Topical Issue "Flowing Matter, Problems and Applications", edited by Federico Toschi, Ignacio Pagonabarraga Mora, Nuno Araujo, Marcello Sega.

** Supplementary material in the form of a .pdf file available from the Journal web page at

https://doi.org/10.1140/epje/i2018-11727-3

a e-mail: mekiel@ippt.pan.pl
}

\section{System, theory and notation}

We consider a point force $\boldsymbol{F}$ at a position $\boldsymbol{r}_{0}$ in a fluid of dynamic viscosity $\mu$. We assume that $\boldsymbol{F}$ generates a fluid flow, and that the fluid velocity $\boldsymbol{u}$ and pressure $p$ satisfy the Stokes equations [36]. We consider the following geometries and the corresponding boundary conditions:

- Unbounded fluid.

- Fluid bounded by a free surface, parallel or perpendicular to $\boldsymbol{F}$. At the surface, the normal component of the fluid velocity $\boldsymbol{u}$ and the normal-tangential components of the stress tensor vanish.

- Fluid bounded by a rigid wall, parallel or perpendicular to $\boldsymbol{F}$. At the wall surface, the fluid velocity vanishes, $\boldsymbol{u}=0$.

\section{Moreover, at infinity $\boldsymbol{u}=0$.}

The classical solutions for a plane free surface, fluidfluid interface and rigid wall are constructed by the method of images [36-40]. The idea is to replace the system - fluid in half-space plus the interface - by infinite fluid plus force multipoles (called "images"), located outside the half-space and chosen in such a way that the same boundary conditions at the interface are satisfied. The position $\boldsymbol{r}_{0}^{\prime}$ of all the images of $\boldsymbol{F}$ is constructed by reflection with respect to the plane of the point $\boldsymbol{r}_{0}$ where $\boldsymbol{F}$ is located. The fluid velocity is a superposition of multipole velocities produced by the point force and its images.

The goal of this work is to provide the visualization of all multipole velocities, in the plane which contains the positions of the point-force and its images, and vector $\boldsymbol{F}$ itself (the plane is perpendicular to the interface). We choose a system of coordinates in which this plane corresponds to the $x y$-plane at $z=0$. Moreover, the $x$-axis is always perpendicular to the force, while the $y$-axis is always parallel 

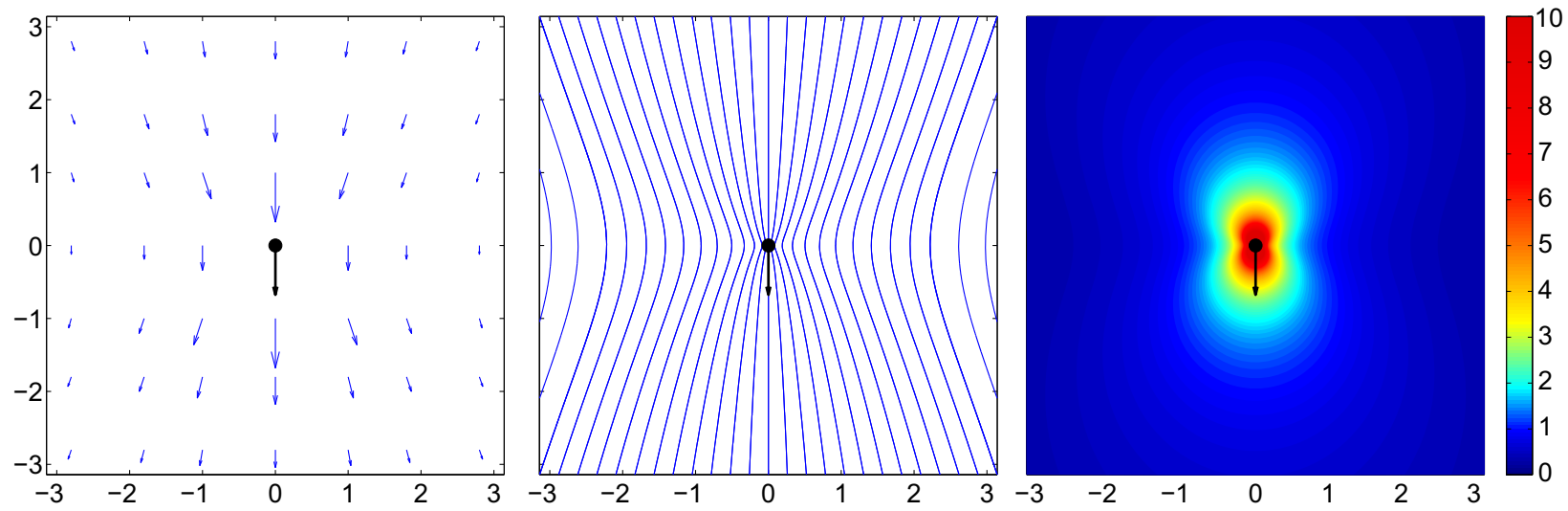

Fig. 1. Fluid velocity around a point force (represented as a black arrow) [36].

to the force, with the opposite sense: $\boldsymbol{F}=(0,-F, 0)$ with $F>0$. as

The relative positions of a given fluid point are denoted

$\mathbf{r}=\left(r_{x}, r_{y}\right)$ from the point force,

$\mathbf{R}=\left(R_{x}, R_{y}\right)$ from its image.

In the following sections, we remind classical expressions [36-42] for the dimensional fluid velocity $\boldsymbol{u}$, together with their derivation, and we provide their graphical representation: vector fields, streamlines and color contour plots of the magnitude (absolute value $|\boldsymbol{u}|$ ). In the Supplemental Material (SM), the horizontal and vertical fluid velocity components are also visualized. We use the distance $h$ from the point-particle to the interface as the length unit, and $v_{0}=\frac{F}{8 \pi \mu h}$ as the velocity unit. For the unbounded fluid, $h$ stands for an arbitrarily chosen length scale (the fundamental solution is scale invariant).

Graphs are plotted based on the following principles. In every plot, the $x$-coordinate is horizontal, and the $y$ coordinate is vertical, and their ranges in every plot are the same, as indicated. The point forces are shown in black and their images in gray. The free surface is denoted as a dashed black line, and the rigid wall as a solid black line. The length of a vector on the velocity field plot represents the magnitude of the velocity (to trace weak velocity fields, enlarge figures in pdf). The vectors are chosen in such a way that they do not overlap and are all scaled relative to the longest vector on the sequence of plots corresponding to the same interface and the same orientation of $\boldsymbol{F}$. The color scale on all the contour plots of velocity magnitude is the same as shown in fig. 1 . Red is chosen for higher velocity values and blue for lower velocity values.

\section{Unbounded fluid}

The fundamental solution of the Stokes equations for the fluid velocity $\left(u_{x}, u_{y}\right)$ generated by a point force $\boldsymbol{F}=$ $(0,-F, 0)$ at $\boldsymbol{r}_{0}=(0,0,0)$ in an unbounded fluid motion- less at infinity is called the Stokeslet and has the form [36]

$$
\begin{aligned}
& u_{x}=(\boldsymbol{T} \cdot \boldsymbol{F})_{x}=-\frac{F}{8 \pi \mu} \frac{r_{x} r_{y}}{r^{3}}, \\
& u_{y}=(\boldsymbol{T} \cdot \boldsymbol{F})_{y}=-\frac{F}{8 \pi \mu}\left(\frac{1}{r}+\frac{r_{y}^{2}}{r^{3}}\right),
\end{aligned}
$$

with $r_{x}=x, r_{y}=y$ and the Oseen tensor $T_{i j}(\boldsymbol{r})=\frac{\delta_{i j}}{r}+$ $\frac{r_{i} r_{j}}{r^{3}}$.

\section{Fluid bounded by a free surface}

\subsection{F parallel to a plane free surface}

For a free surface at $x=0$ and a point force $\boldsymbol{F}=(0,-F, 0)$ at $\boldsymbol{r}_{0}=(h, 0,0)$, the identical image point force $\boldsymbol{F}$ is at $\boldsymbol{r}_{0}^{\prime}=(-h, 0,0)$. With this choice, owing to symmetry, the $x$ component of the resulting fluid velocity and tangentialnormal component of the stress tensor vanish at $x=0$.

Therefore, the fluid velocity, shown in fig. 2, consists of the Stokeslet and the image Stokeslet contributions,

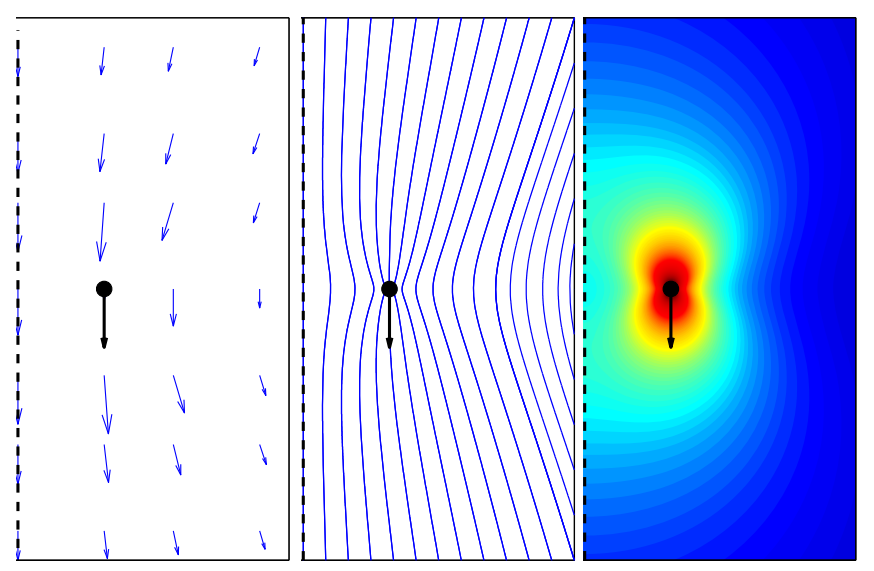

Fig. 2. Fluid velocity around a point force (represented as a black arrow) parallel to a plane free surface - main results. 

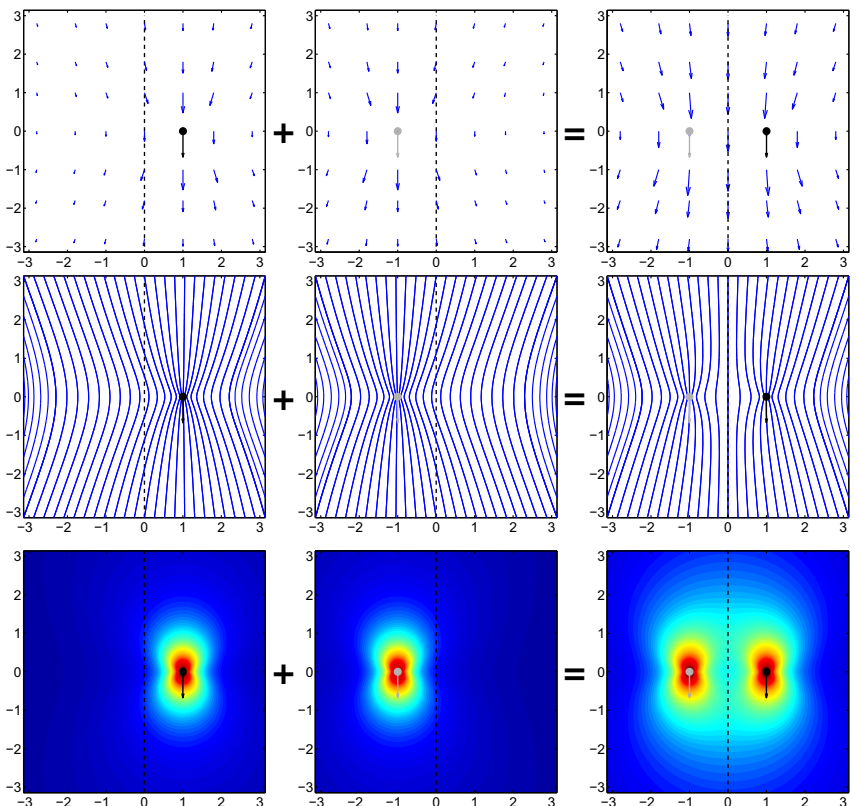

Stokeslet

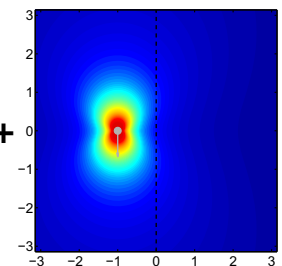

image Stokeslet

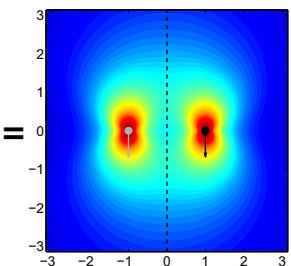

sum

Fig. 3. Fluid velocity around a point force parallel to a plane free surface as a sum of two velocity multipoles.

displayed in fig. 3 and given by the following equations:

$$
\begin{aligned}
& u_{x}=\frac{-F}{8 \pi \mu}(\underbrace{\frac{r_{x} r_{y}}{r^{3}}}_{\text {Stokeslet }}+\underbrace{\frac{R_{x} R_{y}}{R^{3}}}_{\text {image Stokeslet }}), \\
& u_{y}=\frac{-F}{8 \pi \mu}(\underbrace{\frac{1}{r}+\frac{r_{y}^{2}}{r^{3}}}_{\text {Stokeslet }}+\underbrace{\frac{1}{R}+\frac{R_{y}^{2}}{R^{3}}}_{\text {image Stokeslet }}),
\end{aligned}
$$

where for a fluid point $(x, y)$, one has $r_{x}=x-h, R_{x}=$ $x+h, r_{y}=R_{y}=y$. Although the above velocity field is specified in the whole space, the physical meaning is restricted to the right half-space, with $x \geq 0$.

\subsection{F perpendicular to a plane free surface}

For a free surface at $y=0$ and a perpendicular point force $\boldsymbol{F}=(0,-F, 0)$ at $\boldsymbol{r}_{0}=(0,-h, 0)$, the opposite image point force $-\boldsymbol{F}$ is at $\boldsymbol{r}_{0}^{\prime}=(0, h, 0)$. With this choice, owing to symmetry, the $y$ component of the resulting fluid velocity and tangential-normal component of the stress tensor vanish at $y=0$. Therefore, the fluid velocity consists of the Stokeslet and the image Stokeslet contributions, displayed in fig. 5 , and given by the following equations:

$$
u_{x}=\frac{-F}{8 \pi \mu}(\underbrace{\frac{r_{x} r_{y}}{r^{3}}}_{\text {Stokeslet }}-\underbrace{\frac{R_{x} R_{y}}{R^{3}}}_{\text {image Stokeslet }}),
$$

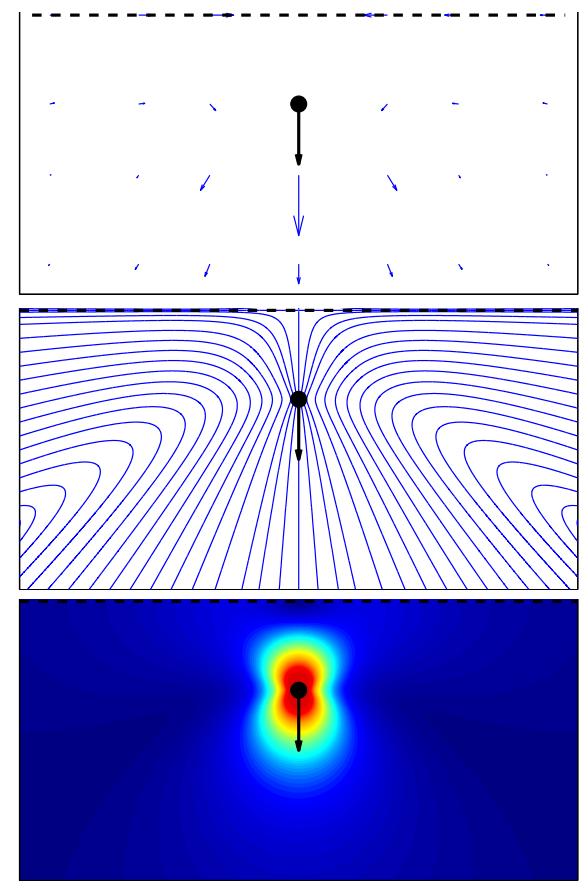

Fig. 4. Fluid velocity around a point force (shown as a black arrow) perpendicular to a plane free surface - main results.
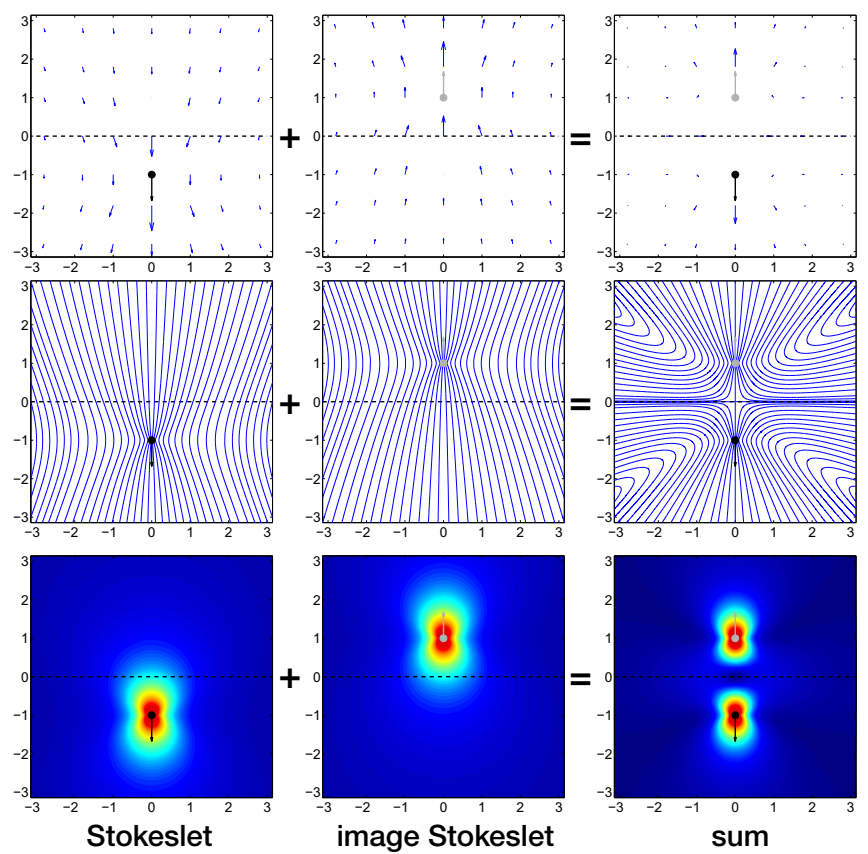

Stokeslet

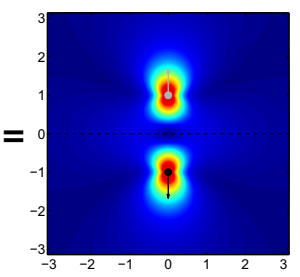

sum

Fig. 5. Fluid velocity around a point force perpendicular to a plane free surface as a sum of two velocity multipoles.

$$
u_{y}=\frac{-F}{8 \pi \mu}(\underbrace{\frac{1}{r}+\frac{r_{y}^{2}}{r^{3}}}_{\text {Stokeslet }}-\underbrace{\frac{1}{R}-\frac{R_{y}^{2}}{R^{3}}}_{\text {image Stokeslet }}),
$$

where for a fluid point $(x, y)$, one has $r_{x}=R_{x}=x$, $r_{y}=y+h, R_{y}=y-h$. Although the above velocity field is specified in the whole space, the physical meaning is restricted to the lower half-space, with $y \leq 0$. 


\section{Fluid bounded by a plane rigid wall}

For a point force in a fluid bounded by a plane rigid wall, the fluid velocity consists of four multipole functions, which satisfy the Stokes equations, decay at infinity as $1 / r, 1 / R, 1 / R^{2}, 1 / R^{3}$ (notice the corresponding scaling of arrows in figures) and are generated by the point force and three image force multipoles. The image Stokeslet is constructed as "reflection" [41,42] of the Stokeslet by the wall, which means that, in the long-distance limit, it cancels the $1 / r$ contribution at the wall when superposed with the Stokeslet. The other two velocity multipoles, the image Stokes doublet and image source doublet, form consecutive "reflections" which cancel the $1 / R^{2}$ and $1 / R^{3}$ contributions at the wall, respectively, and no other terms are left. The image Stokes doublet is Lamb's pressure solid harmonics [36], with the $i$-component $\mathcal{F}_{k j} \partial_{k} T_{i j}$ proportional to the space derivative of the Oseen tensor. The image source doublet, with the $i$-component $f_{k} \partial_{i}\left(R_{k} / R^{3}\right)$, belongs to another family of Lamb's solutions: the fluid velocity is a gradient of harmonic function and the pressure is constant. The force multipoles $\mathcal{F}_{k j}$ and $f_{k}$ will be specified in the following sections.

\subsection{F parallel to a plane rigid wall}

For a rigid wall at $x=0$, and the point force at $\boldsymbol{r}_{0}=$ $(h, 0,0)$, the fluid velocity is shown in fig. 6 . It consists of four velocity multipoles, given by Blake [37,38],

$$
\begin{gathered}
u_{x}=\frac{-F}{8 \pi \mu}(\underbrace{\frac{r_{x} r_{y}}{r^{3}}}_{\text {Stokeslet }} \underbrace{-\frac{R_{x} R_{y}}{R^{3}}}_{\begin{array}{c}
\text { image } \\
\text { Stokeslet }
\end{array}} \\
+\underbrace{\frac{2 h R_{y}}{R^{3}}+\frac{6 h R_{x}^{2} R_{y}}{R^{5}}}_{\begin{array}{c}
\text { image Stokes } \\
\text { doublet }
\end{array}}-\underbrace{\frac{6 h^{2} R_{x} R_{y}}{R^{5}}}_{\begin{array}{c}
\text { image source } \\
\text { doublet }
\end{array}}),
\end{gathered}
$$

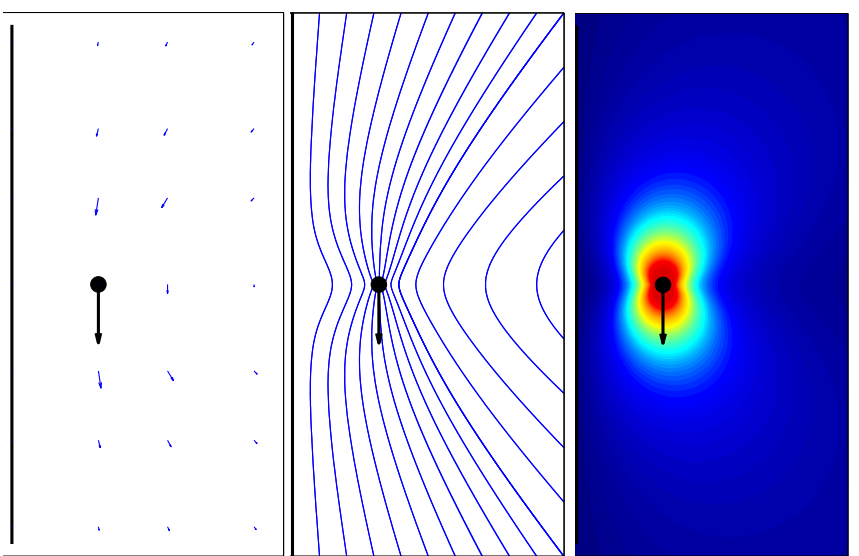

Fig. 6. Fluid velocity around a point force (shown as a black arrow) parallel to a plane rigid wall - main results.
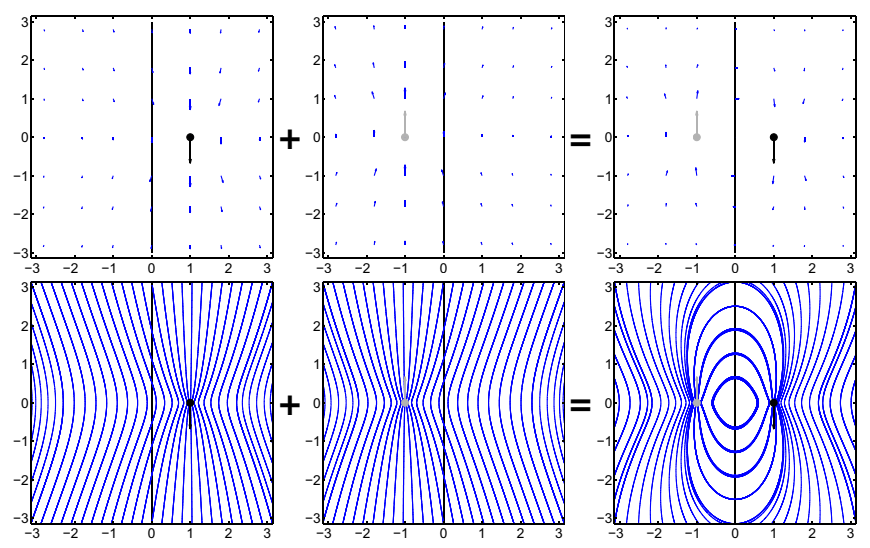

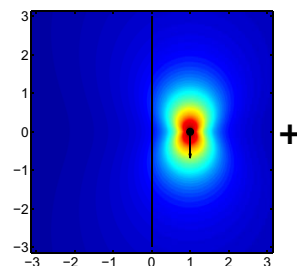

Stokeslet

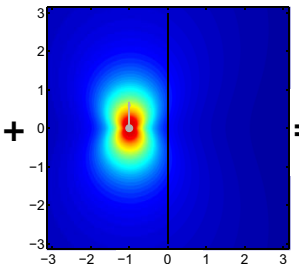

image Stokeslet

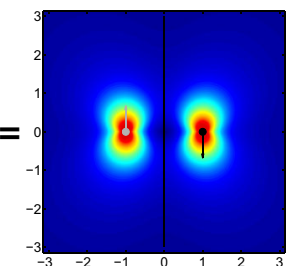

sum 1
Fig. 7. First contribution to the fluid velocity (7), (8): the sum of the Stokeslet and image Stokeslet.
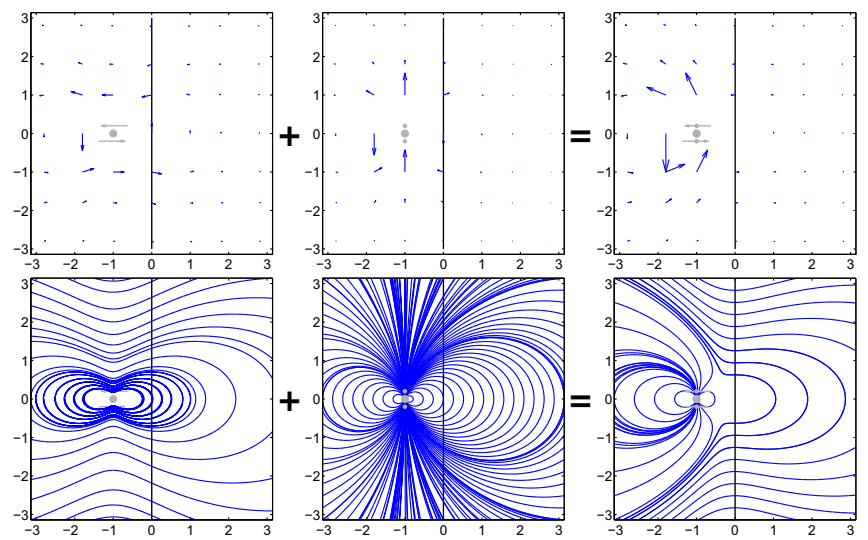

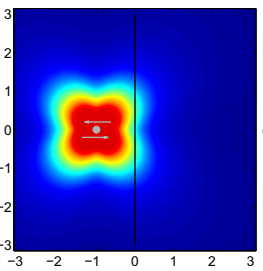

image Stokes

doublet

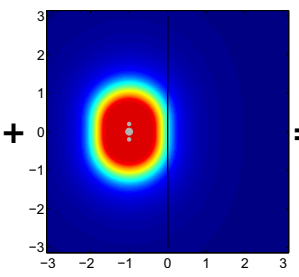

image source
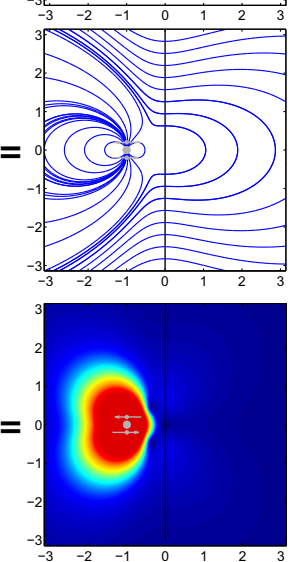

sum 2
Fig. 8. Second contribution to the fluid velocity (7), (8): the sum of the image Stokes doublet and image source doublet.

$$
\begin{aligned}
u_{y}= & \frac{-F}{8 \pi \mu}(\underbrace{\frac{1}{r}+\frac{r_{y}^{2}}{r^{3}}}_{\text {Stokeslet }}-\underbrace{\frac{1}{R}-\frac{R_{y}^{2}}{R^{3}}}_{\begin{array}{c}
\text { image } \\
\text { Stokeslet }
\end{array}} \underbrace{-\frac{2 h R_{x}}{R^{3}}+\frac{6 h R_{x} R_{y}^{2}}{R^{5}}}_{\begin{array}{c}
\text { image Stokes } \\
\text { doublet }
\end{array}} \\
& \underbrace{+\frac{2 h^{2}}{R^{3}}-\frac{6 h^{2} R_{y}^{2}}{R^{5}}}_{\begin{array}{c}
\text { image source } \\
\text { doublet }
\end{array}}),
\end{aligned}
$$



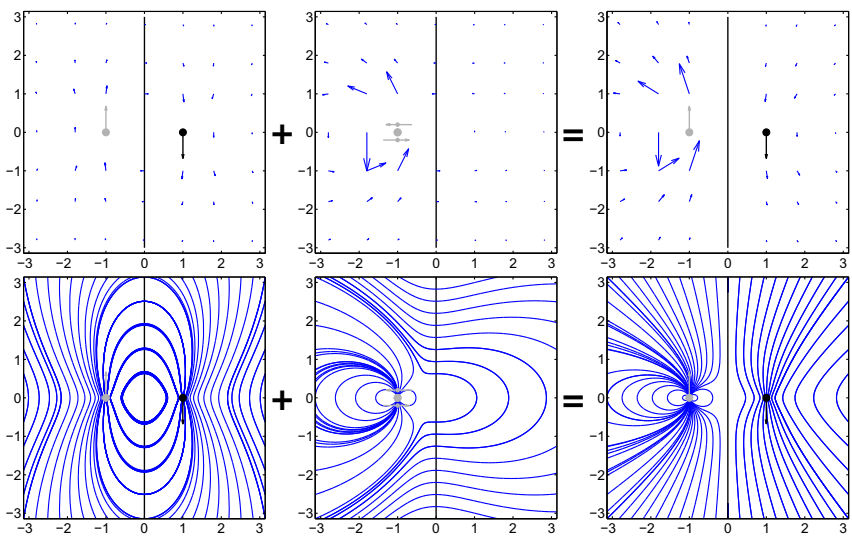

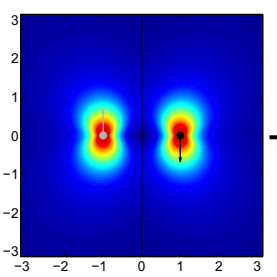

sum 1
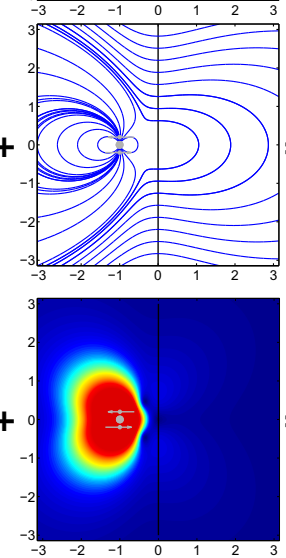

sum 2
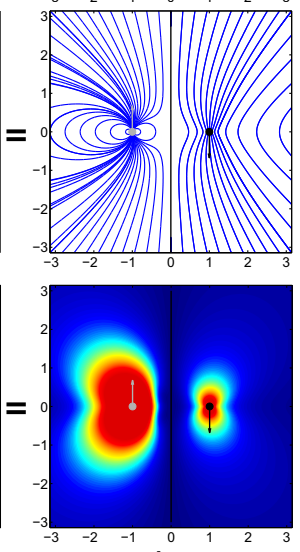

total sum
Fig. 9. Fluid velocity around a point force parallel to a plane rigid wall — sum of figs. 7,8 .

where $r_{x}=x-h, R_{x}=x+h, r_{y}=R_{y}=y$. The image force multipoles are $\boldsymbol{- F}$ for the image Stokeslet, $\mathcal{F}_{k j}=2 h F \delta_{k y} \delta_{j x}$ for the image Stokes doublet and $f_{k}=$ $-2 h^{2} F \delta_{k y}$ for the image source doublet. All velocity multipoles are shown and subsequently summed up in figs. 7-9.

The Stokeslet and the image Stokeslet contributions are displayed in fig. 7 . The image point force $-\boldsymbol{F}$ now has the opposite direction than in case of a free surface. The image potential dipole and the image Stokeslet doublet are shown in fig. 8. The final summation is performed in fig. 9 . Although the velocity field is specified in the whole space, the physical meaning is restricted to the right half-space, with $x \geq 0$.

\section{2 $\mathrm{F}$ perpendicular to a plane rigid wall}

For a rigid wall at $y=0$, and the point force at $\boldsymbol{r}_{0}=$ $(0,-h, 0)$, the image force multipoles are $-\boldsymbol{F}$ for the Stokeslet, $\mathcal{F}_{k j}=-2 h F \delta_{k y} \delta_{j y}$ for the image Stokes doublet and $f_{k}=2 h^{2} F \delta_{k y}$ for the source doublet. The fluid velocity, shown in fig. 10, has the form $[37,38]$,

$$
\begin{aligned}
u_{x}= & \frac{-F}{8 \pi \mu}(\underbrace{\frac{r_{x} r_{y}}{r^{3}}}_{\text {Stokeslet }} \underbrace{-\frac{R_{x} R_{y}}{R^{3}}}_{\begin{array}{c}
\text { image } \\
\text { Stokeslet }
\end{array}}+\underbrace{\frac{2 h R_{x}}{R^{3}}-\frac{6 h R_{x} R_{y}^{2}}{R^{5}}}_{\begin{array}{c}
\text { image Stokes } \\
\text { doublet }
\end{array}} \\
& +\underbrace{\frac{6 h^{2} R_{x} R_{y}}{R^{5}}}_{\begin{array}{c}
\text { image source } \\
\text { doublet }
\end{array}}),
\end{aligned}
$$

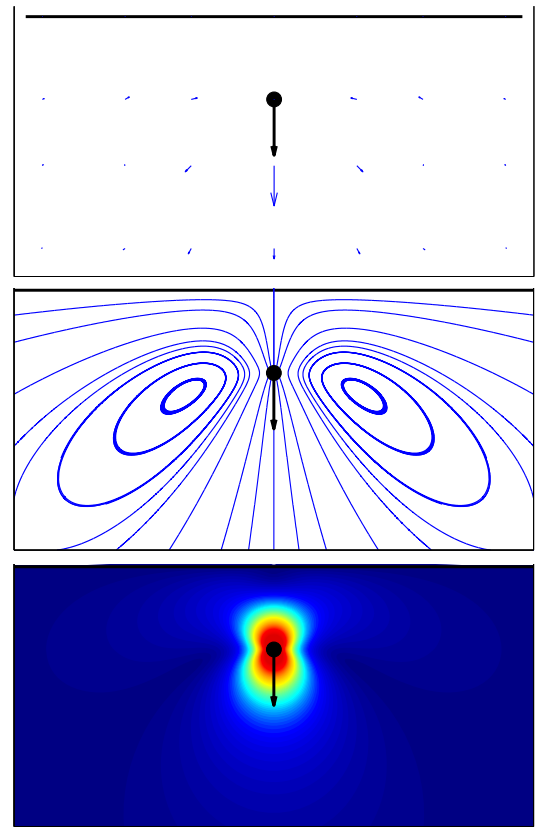

Fig. 10. Fluid velocity around a point force (represented as a black arrow) perpendicular to a plane rigid wall - main results.
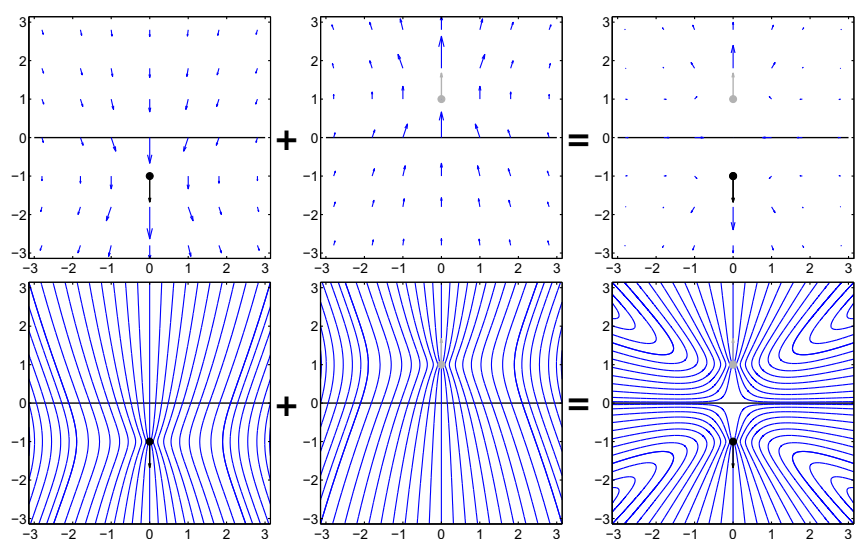

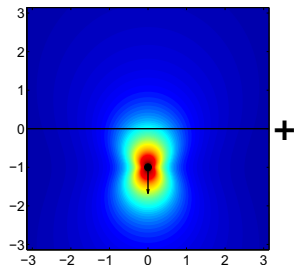

Stokeslet

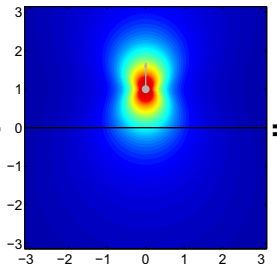

image Stokeslet
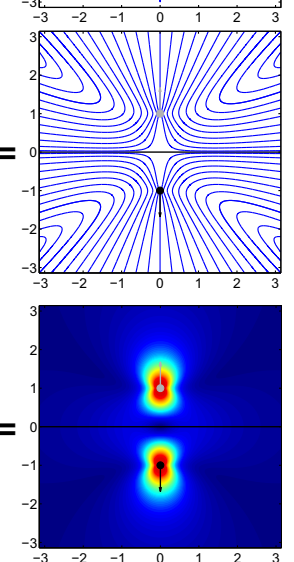

sum 1
Fig. 11. First contribution to the fluid velocity (9), (10): the sum of the Stokeslet and image Stokeslet.

$$
\begin{aligned}
u_{y}= & \frac{-F}{8 \pi \mu}(\underbrace{\frac{1}{r}+\frac{r_{y}^{2}}{r^{3}}}_{\text {Stokeslet }} \underbrace{-\frac{1}{R}-\frac{R_{y}^{2}}{R^{3}}}_{\begin{array}{c}
\text { image } \\
\text { Stokeslet }
\end{array}} \underbrace{+\frac{2 h R_{y}}{R^{3}}-\frac{6 h R_{y}^{3}}{R^{5}}}_{\begin{array}{c}
\text { image Stokes } \\
\text { doublet }
\end{array}} \\
& \underbrace{-\frac{2 h^{2}}{R^{3}}+\frac{6 h^{2} R_{y}^{2}}{R^{5}}}_{\begin{array}{c}
\text { image source } \\
\text { doublet }
\end{array}}),
\end{aligned}
$$




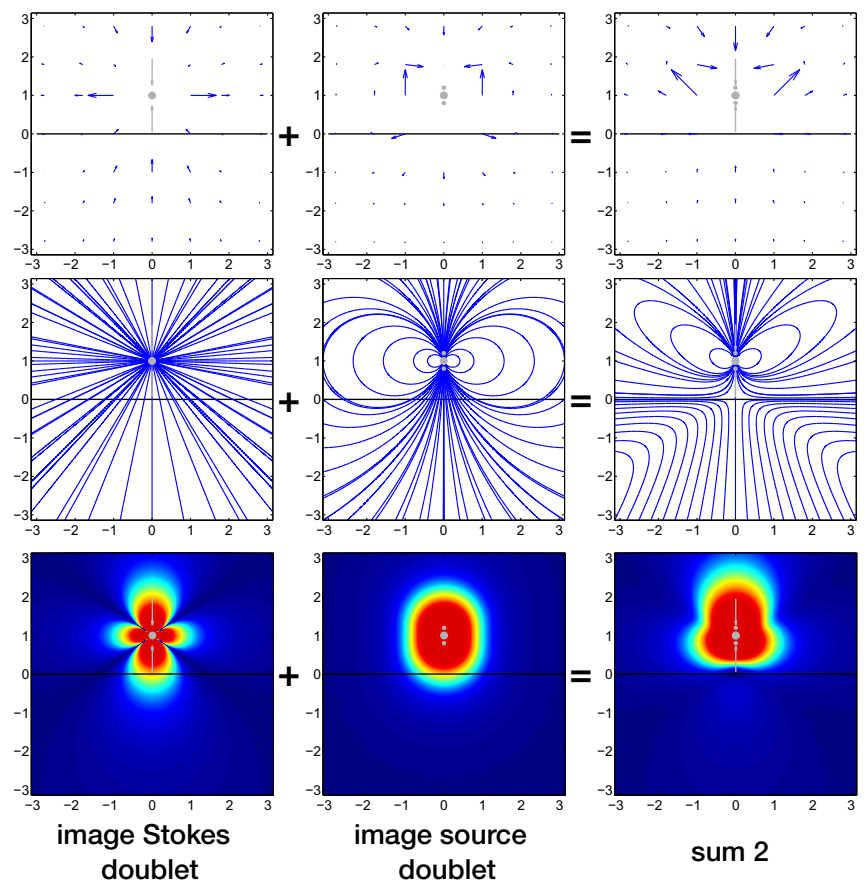

Fig. 12. Second part of the fluid velocity (9), (10): the sum of the image Stokes doublet and image source doublet.

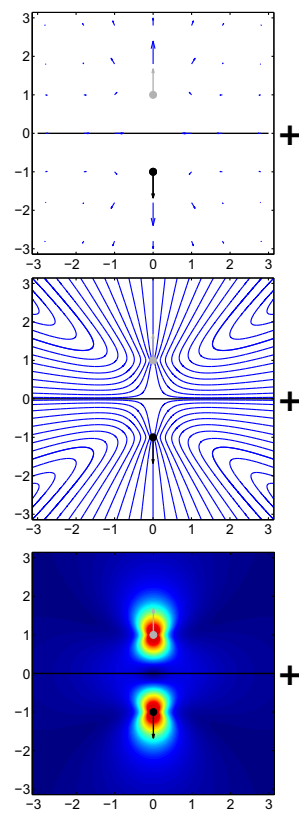

sum 1
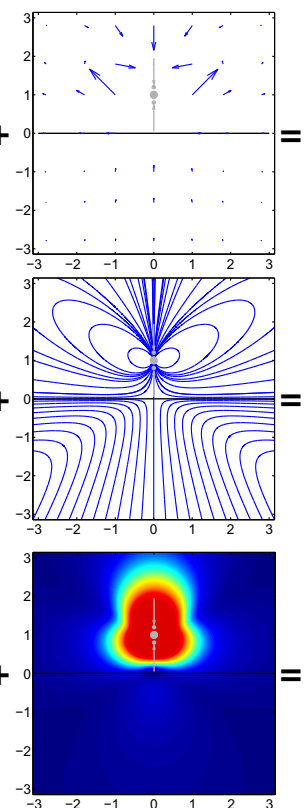

sum 2
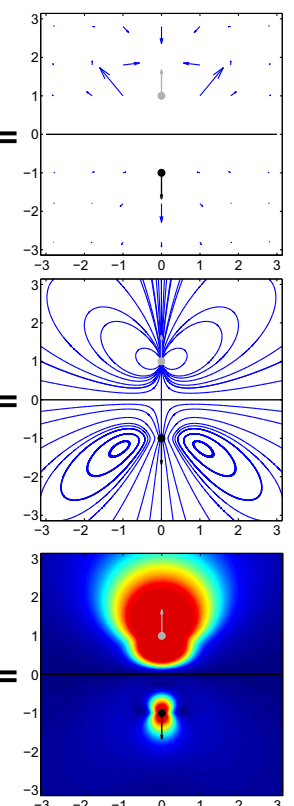

total sum
Fig. 13. Fluid velocity around a point force perpendicular to a plane rigid wall — sum of figs. 11, 12.

where for a given fluid point $(x, y)$, one has $r_{x}=R_{x}=x$, $r_{y}=y+h, R_{y}=y-h$.

All velocity multipoles are shown and subsequently summed up in figs. 11-13. Although the velocity field is specified in the whole space, the physical meaning is restricted to the lower half-space, with $y \leq 0$. The Stokeslet and the image Stokeslet contributions are displayed in fig. 11. The image potential dipole and the image Stokeslet doublet are shown in fig. 12. The final summation is performed in fig. 13.

\section{Conclusions}

Classical Green functions for the Stokes equations in the presence of a plane solid wall, free surface or fluid-fluid interface are still a subject of research [43-45], resulting in useful representations and interpretations. In this paper we contribute to this field by demonstrating the graphical addition of velocity fields generated by different multipoles. It provides a useful quantitative tool to analyze basic features of the fluid flow generated by a point force $\boldsymbol{F}$ close to interface. In particular, it allows to study if higher-order multipoles are significant. For example, we have demonstrated that in case of $\boldsymbol{F}$ perpendicular to a rigid wall, the superposition of the image Stokes doublet and image source doublet is needed to obtain closed streamlines in the vicinity of the point force (see fig. 13).

Moreover, the multipole fluid flows visualized in this paper can be also useful for a graphical representation of the fluid velocity of a particle moving close to an interface. It has been recently shown that even for axisymmetric particles, which are relatively close to a hard wall, a simple analytic approximation for their mobility coefficients can be derived [46].

The graphs presented in this work can be used to analyze basic features of hydrodynamic interactions between a number of micro-objects, or between segments of a single elastic filament, induced by the presence of a plane rigid wall or a plane free surface, such as, e.g., repulsion or attraction, mutual or with respect to the interface $[47,48]$.

The visualization of the fluid flow close to a point force $\boldsymbol{F}$, presented here for a half-space bounded by a free surface or a rigid wall, can be easily generalized for the case of a plane interface between fluids with different dynamic viscosities. The resulting fluid flow velocity in the half-space is a weighted superposition of fluid velocities corresponding to the rigid wall and the free surface, respectively $[39,42,49]$. Denoting as $\mu_{2}$ the viscosity of the fluid on the other side of the interface with respect to $\boldsymbol{F}$, weights of the wall contribution and of the free-surface contribution are given by $\mu_{2} /\left(\mu+\mu_{2}\right)$ and $\mu /\left(\mu+\mu_{2}\right)$, respectively.

This work was supported in part by Narodowe Centrum Nauki under grant No. 2014/15/B/ST8/04359. We acknowledge scientific benefits from COST Action MP1305.

\section{Author contribution statement}

MEJ formulated the idea, designed and supervised the study. The project was originally carried out by RB during an undergraduate training program, as a part of educational activities in microhydrodynamics [50], see also hydro.ippt.gov.pl. The presentation was later redesigned by graduate students MB and MG, and reformulated as a manuscript by MB. MEJ wrote the final version of this work. 
Open Access This is an open access article distributed under the terms of the Creative Commons Attribution License (http://creativecommons.org/licenses/by/4.0), which permits unrestricted use, distribution, and reproduction in any medium, provided the original work is properly cited.

\section{References}

1. M.D. Graham, Annu. Rev. Fluid Mech. 43, 273 (2011).

2. H. Ma, M.D. Graham, Phys. Fluids 17, 083103 (2005).

3. K. Ishimoto, E.A. Gaffney, Phys. Rev. E 88, 062702 (2013).

4. I. Llopis, I. Pagonabarraga, J. Non-Newton. Fluid 165, 946 (2010).

5. W.H. Mitchell, S.E. Spagnolie, J. Fluid Mech. 772, 600 (2015).

6. W.E. Uspal, H.B. Eral, P.S. Doyle, Nat. Commun. 4, 2666 (2013).

7. B. Felderhof, Phys. Fluids 22, 113604 (2010).

8. B. Felderhof, Eur. Phys. J. E 37, 110 (2014).

9. D. Crowdy, S. Lee, O. Samson, E. Lauga, A. Hosoi, J. Fluid Mech. 681, 24 (2011).

10. D. Pimponi, M. Chinappi, P. Gualtieri, C.M. Casciola, Microfluid. Nanofluid. 16, 571 (2014).

11. D. Pimponi, M. Chinappi, P. Gualtieri, C.M. Casciola, J. Fluid Mech. 789, 514 (2016).

12. D. Pimponi, M. Chinappi, P. Gualtieri, Eur. Phys. J. E 41, 28 (2018).

13. J. Elgeti, R.G. Winkler, G. Gompper, Rep. Prog. Phys. 78, 056601 (2015).

14. J. Elgeti, G. Gompper, Eur. Phys. J. ST 225, 2333 (2016).

15. E. Lauga, T.R. Powers, Rep. Prog. Phys. 72, 096601 (2009).

16. E. Lauga, Annu. Rev. Fluid Mech. 48, 105 (2016).

17. S.E. Spagnolie, E. Lauga, J. Fluid Mech. 700, 105 (2012).

18. K. Schaar, A. Zöttl, H. Stark, Phys. Rev. Lett. 115, 038101 (2015).

19. A.P. Berke, L. Turner, H.C. Berg, E. Lauga, Phys. Rev. Lett. 101, 038102 (2008).

20. R. Di Leonardo, D. Dell'Arciprete, L. Angelani, V. Iebba, Phys. Rev. Lett. 106, 038101 (2011).

21. W.R. DiLuzio, L. Turner, M. Mayer, P. Garstecki, D.B. Weibel, H.C. Berg, G.M. Whitesides, Nature 435, 1271 (2005).

22. E. Lauga, W.R. DiLuzio, G.M. Whitesides, H.A. Stone, Biophys. J. 90, 400 (2006).

23. K. Martens, L. Angelani, R. Di Leonardo, L. Bocquet, Eur. Phys. J. E 35, 84 (2012)

24. J.S. Guasto, R. Rusconi, R. Stocker, Annu. Rev. Fluid Mech. 44, 373 (2012).
25. M. Garcia, S. Berti, P. Peyla, S. Rafaï, Phys. Rev. E 83, 035301 (2011).

26. H. Kurtuldu, D. Tam, A. Hosoi, K.A. Johnson, J. Gollub, Phys. Rev. E 88, 013015 (2013).

27. D. Smith, E. Gaffney, J. Blake, J. Kirkman-Brown, J. Fluid Mech. 621, 289 (2009).

28. A. Kumar, M.D. Graham, Soft Matter 8, 10536 (2012).

29. P.M. Vlahovska, D. Barthes-Biesel, C. Misbah, C. R. Phys. 14, 451 (2013).

30. W. Uspal, M.N. Popescu, S. Dietrich, M. Tasinkevych, Soft Matter 11, 434 (2015).

31. R. Dreyfus, J. Baudry, M.L. Roper, M. Fermigier, H.A. Stone, J. Bibette, Nature 437, 862 (2005).

32. S. Martínez-Aranda, F.J. Galindo-Rosales, L. CampoDeaño, Soft Matter 12, 2334 (2016).

33. K. Drescher, R.E. Goldstein, N. Michel, M. Polin, I. Tuval, Phys. Rev. Lett. 105, 168101 (2010).

34. K. Drescher, J. Dunkel, L.H. Cisneros, S. Ganguly, R.E. Goldstein, Proc. Natl. Acad. Sci. U.S.A. 108, 10940 (2011).

35. L. Jibuti, W. Zimmermann, S. Rafaï, P. Peyla, Phys. Rev. E 96, 052610 (2017).

36. S. Kim, S.J. Karrila, Microhydrodynamics: Principles and Selected Applications (Courier Corporation, 2013).

37. J.R. Blake, Math. Proc. Cambridge 70, 303 (1971).

38. J. Blake, A. Chwang, J. Eng. Math. 8, 23 (1974).

39. R. Jones, B. Felderhof, J. Deutch, Macromolecules 8, 680 (1975).

40. G. Perkins, R. Jones, Physica A 171, 575 (1991).

41. H. Lorenz, Zittingsversl. Akad. Wet. 5, 168 (1896).

42. H. Lorenz, Abh. Theor. Phys. 1, 23 (1907).

43. A. Venkatalaxmi, B. Padmavathi, T. Amaranath, Mech. Res. Commun. 31, 465 (2004).

44. Z. Gimbutas, L. Greengard, S. Veerapaneni, J. Fluid Mech. 776, R1 (2015).

45. W. Yan, M. Shelley, arXiv preprint, arXiv:1803.02424 (2018).

46. M. Lisicki, B. Cichocki, E. Wajnryb, J. Chem. Phys. 145, 034904 (2016).

47. T.M. Squires, M.P. Brenner, Phys. Rev. Lett. 85, 4976 (2000).

48. M. Bukowicki, M. Gruca, M.L. Ekiel-Jeżewska, J. Fluid Mech. 767, 95 (2015).

49. J. Bławzdziewicz, M. Ekiel-Jezewska, E. Wajnryb, J. Chem. Phys. 133, 114703 (2010).

50. M.L. Ekiel-Jeżewska, in Human Factors of a Global Society: A System of Systems Perspective, edited by J. Kantola, T. Marek, W. Karwowski, M. Frankowicz, P. Zgaga (CRC Press, 2014) Chapt. 79: Experimenting with teaching contexts, pp. 895-905. 